NASA Technical Memorandum 110160

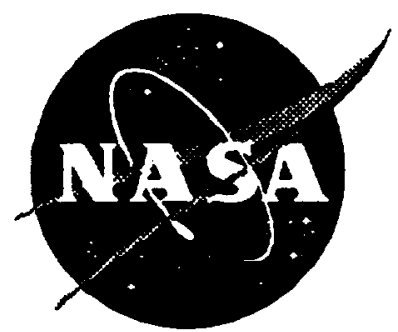

\title{
A Finite Element Propagation Model For Extracting Normal Incidence Impedance In Nonprogressive Acoustic Wave Fields
}

Willie R. Watson

Langley Research Center, Hampton, Virginia

Michael G. Jones

Lockheed Engineering \& Sciences Company, Hampton, Virginia

Sharon E. Tanner and Tony L. Parrott

Langley Research Center, Hampton, Virginia

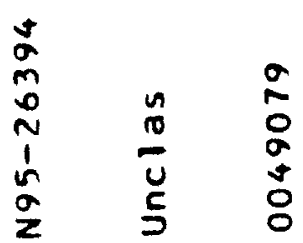

April 1995

National Aeronautics and Space Administration Langley Research Center Hampton, Virginia 23681-0001

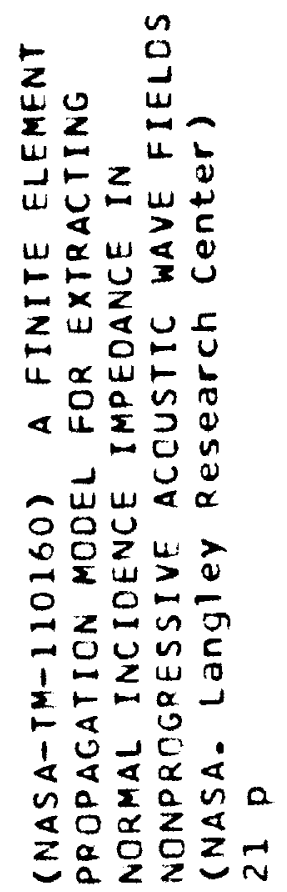




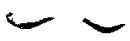




\title{
A Finite Element Propagation Model For Extracting Normal Incidence Impedance In Nonprogressive Acoustic Wave Fields
}

\author{
Willie R. Watson*, Michael G. Jones ${ }^{\ddagger}$, Sharon E. Tanner*, Tony L. Parrott* \\ * NASA Langley Research Center, Hampton, Virginia \\ ${ }^{\ddagger}$ LOCKHEED Engineering \& Sciences Company, Hampton Virginia
}

\begin{abstract}
A propagation model method for extracting the normal incidence impedance of an acoustic material installed as a finite length segment in a wall of a duct carrying a nonprogressive wave field is presented. The method recasts the determination of the unknown impedance as the minimization of the normalized wall pressure error function. A finite element propagation model is combined with a coarse/fine grid impedance plane search technique to extract the impedance of the material. Results are presented for three different materials for which the impedance is known. For each material, the input data required for the prediction scheme was computed from modal theory and then contaminated by random error. The finite element method reproduces the known impedance of each material almost exactly for random errors typical of those found is many measurement environments. Thus, the method developed here provides a means for determining the impedance of materials in a nonprogressive wave environment such as that usually encountered in a commercial aircraft engine and most laboratory settings.
\end{abstract}

\section{Introduction}

The design of increasingly effective and efficient duct treatments for acoustic noise suppression continues to be a critical consideration in the design of environmentally acceptable aircraft propulsion systems. To achieve the full potential of duct treatments in future aircraft engines, it will be necessary to maintain the target impedances of acoustic treatments near their optimum values. A continuing measurement problem in treatment technology is the accurate determination of normal incidence impedance of acoustic material in grazing flow environments. Methods for determining the normal incidence impedance in this environment fall into three categories, "T-tube" method (ref. [1]), in-situ method (refs. [2, 3]), and the propagation model method. The "T-tube" and "in-situ" methods have several drawbacks that are discussed at length in ref. [4]. These two measurement methods do, however, serve as useful complements to the "propagation model" method, which is the subject of this paper.

Propagation model methods for evaluating the acoustic impedance of a material are popular because of their convenience. The conventional method involves measuring the sound attenuation properties in a waveguide lined with the acoustic material over a sufficient length to be effectively infinite. This data is then used with the solution to the wave equation in an infinite waveguide to establish the impedance of the material. The evolution of waveguide 
models for this purpose began over 20 years ago with a uniform mean flow model(ref. [4]). For this case, an analytical expression for the impedance of the material was derived using known transcendental functions and the measured axial wavenumber. Validation of the model in zero flow (i.e., grazing incidence sound only) was achieved by demonstrating that it reproduced the measured normal incidence impedance of a test panel. Waveguide methods were later extended to rectangular ducts with shearing mean flows in one cross sectional direction (refs. [4, 5]). The method presented in ref. [5] was extended to include mean flow shear in two cross sectional directions in ref. [6]. Both the one and two-dimensional sheared flow models developed in refs. [5] and [6] were validated with measured data in ref. [7].

Infinite waveguide models are applicable, in a very straightforward manner, to situations for which a single progressive mode propagates within the waveguide containing the unknown material. However, many conventional liner concepts generate more complex acoustic fields. Thus, measured data must now be interpreted as the superposition of many propagating modes (i.e., multi-modal effects generated by installation of the test specimen and manufacturing tolerances). Broadband liners currently under study contain variable impedance properties and produce multiple modes in the waveguide. The current research effort was motivated by the shortcomings of the current methodology for determining the normal incidence impedance in these more realistic situations. The method developed here uses a propagation model based upon a finite element technique for determining normal incidence impedance from measured wall pressure data. This allows a determination of the impedance of materials in nonprogressive acoustic wave fields contaminated with multi-modal effects and reflections. Although the analysis of this paper assumes a two-dimensional duct without mean flow, it may be extended to three dimensions and to mean flows with shear.

The remainder of this paper is organized into seven sections. The following section 2 describes the physical problem and coordinate system used in the study. Section 3 presents the governing equation and boundary conditions that are solved to obtained the unknown impedance of the acoustic material. Section 4 describes the propagation model (i.e., a linear finite element method). Measured data was not available as input to the model. Therefore, multi-modal analysis was used to simulate the necessary input. This is discussed in section 5 . The unknown impedance of the material is obtained by minimizing the difference between the known and numerically computed wall pressure. The minimization is achieved by a coarse/fine grid search technique in the complex impedance plane. This is the subject of

section 6. Results of impedance predictions for known materials are presented for wave fields containing nonprogressive waves in section 7 . Conclusions relevant to this paper are presented in section 8 .

\section{Description of the Physical Problem}

Figure 1 shows a schematic of the two-dimensional duct used in this study. The amplitudes of right and left moving acoustic waves decay as shown schematically in the figure. The axial and transverse directions are denoted by $x$ and $y$, respectively. The duct is $L$ units long with the source and exit planes located at $x=0$ and $x=L$, respectively. Inputs at the source and exit planes are the source pressure, $p_{s}(y)$, and the normalized exit impedance, $\zeta_{\text {exit }}(y)$, respectively. Throughout this work all impedances are normalized with respect to 
the characteristic impedance of the medium in the duct. The upper wall of the duct is rigid. There are $m$ points located at $x=x_{1}, x_{2}, x_{3} \ldots x_{m}$ along the upper wall, at which the acoustic pressures are known. The sound absorbing material is assumed to be a perforate over honeycomb and constitutes the bottom wall. This material is $L$ units long and is assumed to be point (locally) reacting (i.e. acoustic waves propagate through it normal to the faceplate). The sound absorbing material has an unknown normalized impedance $\zeta(x)$, as shown. The problem at hand is to determine the impedance of the material from the known

data. It should be noted, as suggested by figure 1, that the math model discussed here is limited to a 2-D description which approximates a three-dimensional flow impedance tube. Such flow impedance tube apparatuses can be used to obtain the unknown normal incidence boundary condition from a knowledge of the source pressure, $p_{s}(y)$, exit impedance, $\zeta_{\text {exit }}(y)$, and upper wall pressures. This method of measurement has been traditionally called the "waveguide method". It should be noted that this paper will use analytically based input data to determine the normal incidence impedance, since measured data was not available.

\section{Governing Equation and Boundary Conditions}

Steady-state acoustic pressure waves, propagating within the duct shown in figure 1 satisfy the Helmholtz equation

$$
\frac{\partial^{2} p(x, y)}{\partial x^{2}}+\frac{\partial^{2} p(x, y)}{\partial y^{2}}+k^{2} p(x, y)=0
$$

where $k$ is the free space wavenumber, $k=\frac{2 \pi f}{c}, f$ is the frequency in Hertz, and $c$ is the sound speed in the duct. Before a solution to the acoustic field can be obtained and the unknown impedance extracted, boundary conditions must be prescribed.

Along the source plane of the duct, $x=0$, the acoustic pressure is known

$$
p(0, y)=p_{s}(y)
$$

The boundary condition along the rigid upper wall is equivalent to the requirement that the gradient of acoustic pressure normal to the wall vanishes

$$
\frac{\partial p(x, H)}{\partial y}=0
$$

At the duct termination, $x=L$, the ratio of acoustic pressure to the axial velocity must equal the known exit impedance, $\zeta_{\text {exit }}(y)$

$$
\frac{\partial p(L, y)}{\partial x}=\frac{-i k p(L, y)}{\zeta_{\text {exit }}(y)}
$$

Finally, the lower wall boundary is assumed locally reacting, so that

$$
\frac{\partial p(x, 0)}{\partial y}=\frac{i k p(x, 0)}{\zeta(x)}
$$

When the impedance $\zeta(x)$ is known, equations (1)-(5) constitute a well posed boundary value problem that can be solved to determine the sound field within the duct. Exact solutions to this problem are not available for a general set of input data; therefore, a computational method is required to obtain the solution to equations (1)-(5). 


\section{Duct Propagation Model}

The computational method chosen to solve equation (1), coupled with the boundary condition equations ((2)-(5)), is a Galerkin finite-element method. Details on the method are given in several texts (refs. $[8,9]$ ), and only sufficient detail is presented here for continuity. When applied to the current acoustic problem, the finite-element method may be interpreted as an approximation of the continuous acoustic field as an assemblage of rectangular elements as illustrated in figure 2. Here it is assumed that there are $\mathrm{N}$ nodes in the axial and $\mathrm{M}$ nodes in the transverse directions of the duct. A typical rectangular element, $[I, J]$, is shown in figure 3. Each element consists of four local node numbers labeled 1,2, 3 and 4 , respectively. Each element is considered to have width $a=\left(x_{I+1}-x_{I}\right)$ and height $b=\left(y_{J+1}-y_{J}\right)$ as shown. The objective of the method is to obtain the unknown acoustic pressure at the nodes of each of the $(M-1)(N-1)$ elements.

Galerkin's finite element method is employed to minimize the field error. It should be noted that the field error is distinct from the wall error function, which is used later to extract the unknown impedance. Define the field error function as

$$
E(x, y)=\frac{\partial^{2} p(x, y)}{\partial x^{2}}+\frac{\partial^{2} p(x, y)}{\partial y^{2}}+k^{2} p(x, y)
$$

Within each element $p(x, y)$ is represented as linear combination of four functions, $N_{1}, N_{2}, N_{3}$ and $N_{4}$ which comprise a complete set of basis functions

$$
\begin{array}{rlrl}
p(x, y)=N_{1}(x, y) p_{1} & +N_{2}(x, y) p_{2}+N_{3}(x, y) p_{3}+ & N_{4}(x, y) p_{4} \\
N_{1}(x, y) & =\left[1-\frac{\left(x-x_{J}\right)}{a}\right]\left[1-\frac{\left(y-y_{J}\right)}{b}\right], & & N_{2}(x, y)=\left[\frac{\left(x-x_{J}\right)}{a}\right]\left[1-\frac{\left(y-y_{J}\right)}{b}\right] \\
N_{3}(x, y) & =\left[\frac{\left(x-x_{I}\right)\left(y-y_{J}\right)}{a b}\right], & & N_{4}(x, y)=\left[1-\frac{\left(x-x_{I}\right)}{a}\right]\left[\frac{y-y_{J}}{b}\right]
\end{array}
$$

in which $p_{m}$ is the values of $p(x, y)$ at local node $m$. The variable impedances $\zeta_{\text {exit }}(y)$ and $\zeta(x)$ are represented in a similar manner along each boundary element

$$
\begin{aligned}
\zeta_{\text {exit }}(y) & =\left[\frac{1-\left(y-y_{J}\right)}{b}\right] \zeta_{\text {exit }}\left(y_{J}\right)\left[\frac{y-y_{J}}{b}\right] \zeta_{\text {exit }}\left(y_{J+1}\right) \\
\zeta(x) & =\left[\frac{1-\left(x-x_{I}\right)}{a}\right] \zeta\left(x_{I}\right)+\frac{\left(x-x_{I}\right)}{a} \zeta\left(x_{I+1}\right)
\end{aligned}
$$

In an ideal sense, the solution to the sound field is obtained when the field error, $E(x, y)$, is identically zero at each point of the domain. This is approximately achieved by requiring that the field error function be orthogonal to each basis function $N_{m}(x, y)$. Contributions to the minimization of the field error function from a typical element are

$$
\int_{x_{I}}^{x_{I+1}} \int_{y_{J}}^{y_{J+1}} E N_{I} d y d x=\int_{x_{I}}^{x_{I+1}} \int_{y_{J}}^{y_{J+1}}\left[\frac{\partial^{2} p^{[I, J]}}{\partial x^{2}}+\frac{\partial^{2} p^{[I, J]}}{\partial y^{2}}+k^{2} p^{[I, J]}\right] N_{I} d y d x
$$

The second derivative terms in equation (10) are integrated by parts in order that the linear basis functions can be used

$$
\begin{aligned}
& \int_{x_{I}}^{x_{I+1}} \int_{y_{J}}^{y_{J+1}} E N_{I} d y d x=-\int_{x_{I}}^{x_{I+1}} \int_{y_{J}}^{y_{J+1}}\left[\frac{\partial p^{[I, J]}}{\partial x} \frac{\partial N_{I}}{\partial x}+\frac{\left.\partial p^{[I, J}\right]}{\partial y} \frac{\partial N_{I}}{\partial y}-k^{2} p^{[I, J]} N_{I}\right] d y d x \\
& +\int_{y_{J}}^{y_{J+1}}\left[\frac{\partial \boldsymbol{p}^{[I, J]}(L, y)}{\partial x} N_{I}(L, y)-\frac{\left.\partial p^{[I, J}\right]_{(0, y)}}{\partial x^{\prime}} N_{I}(0, y)\right] d y \\
& +\int_{x_{I}}^{x_{I+1}}\left[\frac{\partial p^{[I, J]}{ }_{(x, H)}}{\partial y} N_{I}(x, H)-\frac{\left.\partial^{[I, J]}\right]_{(x, 0)}}{\partial y} N_{I}(x, 0)\right] d x
\end{aligned}
$$


Substituting the wall and exit boundary condition into the line integrals in (11) gives

$$
\begin{aligned}
\int_{x_{I}}^{x_{I+1}} \int_{y_{J}}^{y_{J}+1} E N_{I} d y d x= & -\int_{x_{I}}^{x_{I+1}} \int_{y_{J}}^{y_{J+1}}\left[\frac{\partial p^{[I, J]}}{\partial x} \frac{\partial N_{I}}{\partial x}+\frac{\partial p^{[I, J]}}{\partial y} \frac{\partial N_{I}}{\partial y}-k^{2} p^{[I, J]} N_{I}\right] d y d x \\
& +\int_{y_{J}}^{y_{J+1}}\left[\frac{i k p(I, J)}{\zeta_{e I i t}(L, y)} N_{I}(L, y)+\frac{\partial p^{[I, J]}(0, y)}{\partial x} N_{I}(0, y)\right] d y \\
& -i k \int_{x_{I}}^{x_{I+1}}\left[\frac{p^{[I, J]}(x, 0)}{\zeta(x)} N_{I}(x, 0)\right] d x
\end{aligned}
$$

where the line integrals in equation (12) are evaluated only for elements which lie along the boundary of the duct. The contribution to the minimization of the field error for each element is expressed in matrix form as

$$
\int_{x_{J}}^{x_{I+1}} \int_{y_{J}}^{y_{J+1}} E N_{I} d y d x=\left[A^{[I, J]}\right]\left\{\Phi^{[I, J]}\right\}
$$

where $\left[A^{[I, J]}\right]$ is a $4 \times 4$ complex matrix for each element $[I, J]$, and $\left\{\Phi^{[I, J]}\right\}$ is a $4 \times 1$ column vector containing the unknown acoustic pressure at the four nodes of the element. The coefficients in the local stiffness matrix, $\left[A^{[I, J]}\right]$, were computed in closed form.

Assembly of the global equations for the computational domain is a basic procedure in the finite element method. Appropriate shifting of rows and columns is all that is required to add the local element matrix, $\left[A^{[I, J]}\right]$, directly into the global matrix, $[A]$. Assembling the elements for the entire domain results in a matrix equation of the form:

$$
[A]\{\Phi\}=\{F\}
$$

where $[A]$ is a complex matrix whose order is $M N$, and $\{\Phi\}$ and $\{F\}$ are $M N \times 1$ column vectors. The vector $\{\Phi\}$ contains the nodal values of the unknown acoustic pressure and $\{F\}$ is the zero vector. It is necessary to apply the source pressure condition to this system of equations before a solution can be obtained. Satisfying the noise source boundary condition consists simply of setting all nodal values of acoustic pressure at the source plane $(x=0)$ to the known value of source pressure, $p_{s}(y)$. Thus inserting these conditions into the assembled global matrix equation (14), introduces nonzero elements into the first $2 M$ components of $\{F\}$. Further details on imposing source conditions are described elsewhere (refs. $[8,9]$ ).

The global matrix $[A]$ generated by Galerkin's Method following application of the source conditions is a complex matrix. Fortunately, owing to the discretization scheme used, it will also be block tridiagonal. The structure of matrix $[A]$ prior to imposing boundary conditions is shown in figure 4 , where the superscript $T$ denotes matrix transpose. Note that $[A]$ is a square symmetric block tridiagonal matrix whose order is $M N$. This global matrix contains a number of major blocks $\left(A_{I}, B_{I}\right)$ which are themselves square and tridiagonal as shown in the figure. The diagonal major blocks, $A_{I}$ are also symmetric. Much practical importance arises from this symmetric structure as it is convenient for minimizing storage and maximizing computational efficiency. Special matrix techniques exist for a solution of this structure following application of source conditions ${ }^{1}$. All computation and storage is performed only on the lower triangular portion of the matrix $[A]$.

\footnotetext{
${ }^{1}$ Gaussian elimination with partial pivoting and equivalent row infinity norm scaling is used to reduce the rectangular system to upper triangular form. Back substitution is then employed to obtain the solution for the acoustic pressure at the $N M$ node points
} 


\section{Data Input to Duct Propagation Model}

Three sets of boundary data are required in addition to the rigid upper wall condition, in order for the duct propagation model to uniquely determine the upper wall pressure. The foregoing equations make use of this unique relationship between the upper wall pressures, $p\left(x_{I}, H\right)$, and the following three sets of data

1. The source plane pressure, $p_{s}(y)$

2. The exit plane impedance, $\zeta_{\text {exit }}(y)$

3. The lower wall impedance function, $\zeta(x)$

If any two and the upper wall pressures are known, the remaining can be determined. Here we are seeking the unknown impedance function of the lower wall, $\zeta(x)$. It will be determined by specifying the upper wall pressures, the source pressure and exit impedance.

Experimental data were not available for input to the finite element duct propagation model. Thus, in this effort, we assume a uniform liner impedance of the bottom wall, and use multi-modal analysis to determine the upper wall pressures, the source pressure, and exit impedance. To begin, the conventional modal solution in the duct of figure 1, for a constant impedance at the lower wall, is

$$
\begin{gathered}
p(x, y)=\sum_{n=1}^{n \text { modes }}\left[A_{n} e^{-i k_{n} x}+B_{n} e^{i k_{n} x}\right] p_{n}(y) \\
p_{n}(y)=\cos \left(\lambda_{n} y\right)+\tan \left(\lambda_{n} H\right) \sin \left(\lambda_{n} y\right) \\
k_{n}^{2}=k^{2}-\lambda_{n}^{2} \\
\zeta=\frac{i k H}{\lambda_{n} H \tan \left(\lambda_{n} H\right)}
\end{gathered}
$$

Here, nmodes is the number of modes, $A_{n}$ and $B_{n}$ are the chosen mode amplitude coefficients of the right and left moving acoustic waves in the duct, respectively, and the eigenvalues, $\lambda_{n}$, are obtained by solving the transcendental equation (18).

The source pressure and exit impedance used as input data here, are obtained by substituting the series in equation (15) (i.e., with chosen values of $A_{n}, B_{n}$ and nmodes) into equations (2) and (4), respectively, to obtain

$$
\begin{gathered}
p_{s}(y)=\sum_{n=1}^{n m o d e s}\left[A_{n}+B_{n}\right] p_{n}(y) \\
\zeta_{\text {exit }}(y)=\frac{-k \sum_{n=1}^{n \text { modes }}\left[A_{n} e^{-i k_{n} L}+B_{n} e^{i k_{n} L}\right] p_{n}(y)}{\sum_{n=1}^{n \text { modes }} k_{n}\left[B_{n} e^{i k_{n} L}-A_{n} e^{-i k_{n} L}\right] p_{n}(y)}
\end{gathered}
$$

In order to obtain the known upper wall pressure that is required to extract the unknown impedance, the series in (15) is evaluated at the $m$ axial locations along the upper wall

$$
p\left(x_{I}, H\right)=\sum_{n=1}^{n \text { modes }}\left[A_{n} e^{-i k_{n} x_{I}}+B_{n} e^{i k_{n} x_{I}}\right] p_{n}(H)
$$




\section{Extraction of the Unknown Impedance}

The goal of the impedance extraction method described in this work is to determine the unknown impedance, $\zeta(x)$, of an acoustic material from the data input. The procedure is to numerically determine the impedance function $\zeta(x)$, such that the pressure along the top wall reaches its known value at each of the $m$ points. The procedure consists of repeatedly cycling through the solution to the boundary value problem (equations (1)-(5)), and obtaining a set of upper wall pressures for each impedance function. As each new set of wall pressures is computed, it is compared to the known values until convergence is achieved. Convergence of the procedure is guaranteed, since the boundary value problem is well-posed.

The idea is best illustrated by considering a constant impedance, $\zeta$. We define the unknown impedance as

$$
\zeta=\theta+i \chi
$$

where $\theta$ is the resistance and $\chi$ the reactance. Resistance values are positive whereas reactance values span the real axis

$$
0 \leq \theta \leq \infty, \quad-\infty \leq \chi \leq \infty
$$

It should be apparent that searching the entire upper half plane of the resistance/reactance space for the unknown impedance is impractical. Thus, we introduce the tranformation

$$
\chi=\cot (k d), \quad 0 \leq k d \leq \pi
$$

and search for the unknown impedance in the $(\theta, k d)$ plane, where $\theta$ is limited to $0 \leq \theta \leq \theta_{\text {max }}$. Rules for selecting $\theta_{\max }$ will be discussed later. It should be noted that equations (22) and (24) represent the impedance model for many perforates over honeycomb used in current aircraft engines and the parameter $d$ is the backing depth of the perforate (ref. [10]).

We now divide the complex plane $(\theta, k d)$ into IMAX evenly spaced intervals in the $\theta$ direction and $J M A X$ evenly spaced points in the $k d$ direction, as shown in figure 5 . The increment spacing $\Delta \theta$ and $k \Delta d$ are

$$
\Delta \theta=\frac{\theta_{\max }}{I M A X-1}, \quad k \Delta d=\frac{\pi}{J M A X-1}
$$

Thus a point $\zeta_{I J}$ in the uniform impedance grid is

$$
\zeta_{I J}=\theta_{I}+i \chi_{J}, \quad \theta_{I}=(I-1) \Delta \theta, \quad \chi_{J}=\cot (J-1) k \Delta d
$$

We will establish a rule of thumb for determining $I M A X$, and $J M A X$ in the next section. We now define the global normalized wall error function at a point $(I, J)$ in the impedance plane. Let $\zeta$ denote the impedance of the unknown material. If the known upper wall pressures corresponding to $\zeta$ are $\bar{p}\left(x_{n}, H\right)$ and those computed from the finite element solution with $\zeta_{I J}$ are $p\left(x_{n}, H\right)$, then a measure of the closeness of $\zeta_{I J}$ to $\zeta$ is given by the normalized wall error function, $E W\left(\zeta_{I J}\right)$

$$
E W\left(\zeta_{I J}\right)=\frac{\overline{E W}\left(\zeta_{I J}\right)}{\bar{E}_{\max }}
$$




$$
\overline{E W}\left(\zeta_{I J}\right)=\frac{1}{m} \sum_{n=1}^{m}\left|\bar{p}\left(x_{n}, H\right)-p\left(x_{n}, H\right)\right|
$$

in which || denotes the absolute value of a complex quantity, $\bar{E}_{\max }$ is the maximum value of $\overline{E W}$ for all points $\zeta_{I J}$ in the impedance grid, and $m$ is the number of known wall pressures.

Determining the unknown impedance of the material is now recast as a minimization problem. Thus, $\zeta$ should be chosen such that $E W(\zeta)$ is a global minimum. The global minimum is obtained using a two-step method. First, we use a coarse grid in the impedance plane and tabulate the normalized wall error function to determine the location in that grid of the minimum point $\left(\theta^{1}, k d^{1}\right)$. We use a fine grid centered about $\left(\theta^{1}, k d^{1}\right)$, where $\Delta \theta$ and $\Delta k d$ are now much smaller. The location of the minimum point of the fine grid corresponds to the unknown impedance.

\section{Results}

A computer code implementing the impedance extraction method has been developed. The finite element matrix equation (14) is solved using a routine from the highly developed software package "Lapack,"(ref. [11]) and minimization of the normalized wall error function is performed internally by an in-house computer code. The unknown impedance, $\zeta$, is returned by the in-house code. Results were computed using a Dec-Alpha work station and were not computationally intensive (i.e., requiring only 0.5 seconds of CPU time for each point in the impedance grid). In this section, the integrity of the impedance extraction method is tested on three materials for which the impedance, $\zeta$, is known. The first two are materials for which $\zeta=1+1 i$ and $\zeta=3+2 i$, respectively. The last material is a rigid wall, for which the impedance approaches $\infty+\infty i$, which corresponds to an admittance of, $0+0 i$. Thus, for convenience, analysis of the rigid wall case is conducted in the admittance plane.

Input data required to extract the impedance of each liner was obtained by solving equation (18) with the known $\zeta$ and calculating the source pressure and exit impedance from equation (19) and (20), respectively. Equation (21) was then evaluated at $m$ evenly spaced locations to provide the known wall pressure for the wall error function. In an attempt to determine the effects of error in the input data, a number of cases were run with the upper wall pressure distribution randomly perturbed according to

$$
\hat{p}\left(x_{I}, H\right)=p\left(x_{I}, H\right) E_{\tau}
$$

where $\hat{p}\left(x_{I}, H\right)$ is the perturbed pressure and $E_{r}$ is the random error. For the cases presented in this report, the range of random error was set to $\pm 0.1 \mathrm{~dB}$, so that

$$
E_{r}=1 \pm N_{r} 10^{0.1 / 20}
$$

where $N_{r}$ is a random number between 0 and 1 . The $\pm 0.1 \mathrm{~dB}$ random error range was not arbitrarily chosen, but is typical of that experienced in the Langley Grazing Flow Impedance Tube Facility. For each material, impedance predictions are presented for a single mode nonprogressive wave field (nmodes $=1, A_{1}=1.0, B_{1}=0.5$ ). The duct geometry for which calculations were made was chosen to be that of the Langley Flow Impedance Tube Facility (i.e., $H=2.0$ inches, $L=23.0$ inches) test section. Results are presented for two source 
frequencies, $\mathrm{f}=500 \mathrm{Hertz}$ and $\mathrm{f}=3,000 \mathrm{Hertz}$. A $231 \times 21$ evenly spaced grid is used $(N=231$ and $M=21$ ) in the finite element discretization for all calculations. This grid ensured that a minimum of ten elements per wavelength was used in the finite element discretization at the highest frequency of interest for each of the wave fields considered.

Numerical experimentation has shown that a $51 \times 31$ uniform grid (i.e., $I M A X=51$ and $J M A X=31$, with $\Delta \theta=k \Delta d=0.1$ ) is typically sufficient for the coarse grid search procedure. Note that while this grid covers nearly all possible reactance values, the resistance only ranges from 0 to 5 (i.e., $\theta_{\max }=5$ ). If larger values of resistance are expected, a larger value of $\theta_{\max }$ should be used. After the coarse grid procedure has been completed, a fine grid search is conducted. Again, numerical experimentation has shown that a $21 \times 21$ uniformly spaced fine grid (i.e., $I M A X=21$ and $J M A X=21$, with $\Delta \theta=k \Delta d=0.01$ ) is sufficient for convergence to the unknown impedances.

Convergence of the impedance prediction method is best illustrated using contour plots in the $(\theta, \chi)$ plane. Figure 6 shows contour plots of $E W(\zeta)$ for the fine grid at a frequency of 500 Hertz. Ten evenly spaced points (i.e., $m=10$ ) were used to construct the wall error function. The known impedance is $\zeta=1+1 i$, and the resistance and reactance are plotted on the horizontal and vertical axes, respectively. The coarse grid contours collapse to a single point at $\zeta=1.00+0.97 i$. As can be seen in the figure 6 , the global minimum point of $E W(\zeta)$ for the fine grid lies within the contour labeled 7 . Thus the returned impedance is the value at the grid point closest to the center of that contour, $\zeta=1.00+0.99 i$. It should be noted that separate tests were conducted to show that the error is even smaller at $\zeta=1+1 i$, since the fine grid used as a standard in this study did not include this particular impedance as a point in the grid.

Figure 7 presents a comparison of the known resistance and reactance for the first material $(\theta=\chi=1)$ with the predicted values, for input data with and without random error. The two plots in the upper half of the figure were computed at a frequency of 500 Hertz, while the two in the lower half of the figure were computed at 3,000 Hertz. The independent variable for the horizontal axis is the number of evenly spaced input wall pressure points, $m$, used to determine the wall error function. Results are shown for $m=5,10,46$ and 230 . Note that the impedance prediction method does an excellent job of determining the normal incidence resistance and reactance, with and without random error. Predicted resistance values are slightly less accurate at the higher frequency for small values of $m$. Collectively, these graphs show that the predicted impedance is independent of $m$. Figure 8 shows similar results for the second material, whose known resistance and reactance was $\theta=3$, and $\chi=2$, respectively. Overall trends are consistent with that of figure 7 . The largest error (i.e., approximately $4 \%$ ) occurs in the reactance prediction at 3,000 Hertz for $m=5$ when there is random error.

The third and final material was a rigid wall, which was included in this study in an attempt to cover the realistic range for the majority of grazing incidence impedance measurements. Since the impedance for a rigid wall approaches $\zeta=\infty+\infty i$, it was not feasible to perform an impedance plane grid search to try to determine the appropriate normal incidence impedance. However, the known admittance, $\beta=1 / \zeta=\kappa+i \sigma$, for this case is zero $(\beta=0+0 i)$. For this reason, an admittance plane grid search was performed for this material. A coarse grid search was conducted with $\Delta \kappa=\Delta \sigma=0.1$, over ranges of $0 \leq \kappa \leq 2$ and $-1 \leq \sigma \leq 1$. A fine grid search was then conducted with $\Delta \kappa=\Delta \sigma=0.01$, over ranges of 
$0 \leq \kappa \leq 0.2$ and $-0.1 \leq \sigma \leq 0.1$. The results are shown in figure 9. Predicted admittances are in exact agreement with the known value for each frequency, with and without random error in the input data.

Impedance predictions were also obtained for each of the two soft materials, but for the following sound fields

1. A single mode progressive wave field (nmodes $=1, A_{1}=1.0, B_{1}=0.0$ )

2. A multi-modal wave field without reflections ( $n$ modes $=2, A_{1}=A_{2}=1.0, B_{1}=B_{2}=0.0$ )

3. A multi-modal wave field with significant reflections ( $n$ modes $=2, A_{1}=A_{2}=1.0, B_{1}=0.5, B_{2}=0.0$ )

Graphical results for these three fields are not presented for the sake of brevity. However, it was observed that impedance predictions were in good agreement to the known impedances using each of these three sound fields. In fact, when the wall pressure was not subjected to random error, predicted impedances for each sound field was identical to that obtained for the single mode nonprogressive wave field (see figures 7, 8, and 9). Studies were also performed for larger random errors. When the random error was increased to a level of $5 \mathrm{~dB}$ (well above those typically experienced in normal applications), a weak dependence of the error in the prediction versus the number of wall pressure points was observed. This dependence was determined to be a decreasing function of $m$. Thus, as should be expected, an increasing number of wall pressure points should be used to increase the accuracy in the predictions for measurement systems with larger random errors.

\section{Conclusions}

A coarse/fine grid impedance plane search technique has been developed for extracting the unknown impedance of an acoustic material. A main advantage of the method is that it is applicable to a nonprogressive wave environment, such as that usually encountered in commercial aircraft engines and most laboratory settings. Although the method as presented here is restricted to two-dimensional ducts without mean flow, it may be extended to three dimensions and to mean flows with shear. Data input for the predictions presented in this paper were obtained from modal theory, but this data could be replaced with measurements taken in a grazing flow impedance tube with the test specimen installed. Results of this study show that the method is extremely effective in extracting the impedance of a known material in complicated nonprogressive wave fields. When there is significant random error in the input data, a large number of wall data points are required for an accurate impedance prediction. The method is quite insensitive to random error typical of that found in most high quality measurement systems. The method has been found to be a simple and powerful tool for analytically based input data. There is now a need to test the method with measured data. 


\section{References}

[1] Feder, E.; and Dean, III, L.W.: "Analytical and Experimental Studies for Predicting Noise Attenuation in Acoustically Treated Ducts for Turbofan Engines," NASA CR1373 (Sept. 1969).

[2] Phillips, B.: "Effects of High Value Wave Amplitude and Mean Flow on a Helmholtz Resonator," NASA TMX-1582 (May 1967).

[3] Phillips, B.; and Morgan, C. J.: "Mechanical Absorption of Acoustic Oscillations in Simulated Rocket Combustion Chambers," NASA TN D-3792 (Jan. 1967).

[4] Armstrong, D. L.; and Olsen, R. F.: "Impedance Measurements of Acoustic Duct Liners With Grazing Flow," Boeing paper presented at the 87th Meeting of the Acoustical Society of America (New York, NY), April 1974.

[5] Watson, Willie R : "A Method for Determining Acoustic-Liner Admittance in a Rectangular Duct With Grazing Flow From Experimental Data," NASA TP-2310, 1974.

[6] Watson, Willie R: "A New Method for Determining Acoustic-Liner Admittance in Duct With Sheared Flow in Two Cross-Sectional Directions," NASA TP-2518, 1985.

[7] Parrott, Tony L., Watson, Willie R, and Jones Michael G. : "Experimental Validation of a Two-Dimensional Shear-Flow Model for Determining Acoustic Impedance," NASA TP-2679, 1987.

[8] Zienkiewicz, O. C.: "The Finite Element Method In Engineering Science," McGraw-Hill Book Company, London, 1971.

[9] Desai, Chandrakant S.; and Abel, John F.: "Introduction To The Finite Element Method," Van Nostrand Reinhold Company, New York, N. Y. 1972.

[10] Kraft, R.E.: "Theory and Measurement of Acoustic Wave Propagation In MultiSegmented Rectangular Ducts," P.h.D. Thesis, University of Cincinnati, 1976.

[11] Anderson, E. et al., " $L A P A C K$ user's guide", Society for Industrial and Applied Mathematics, 1992. 


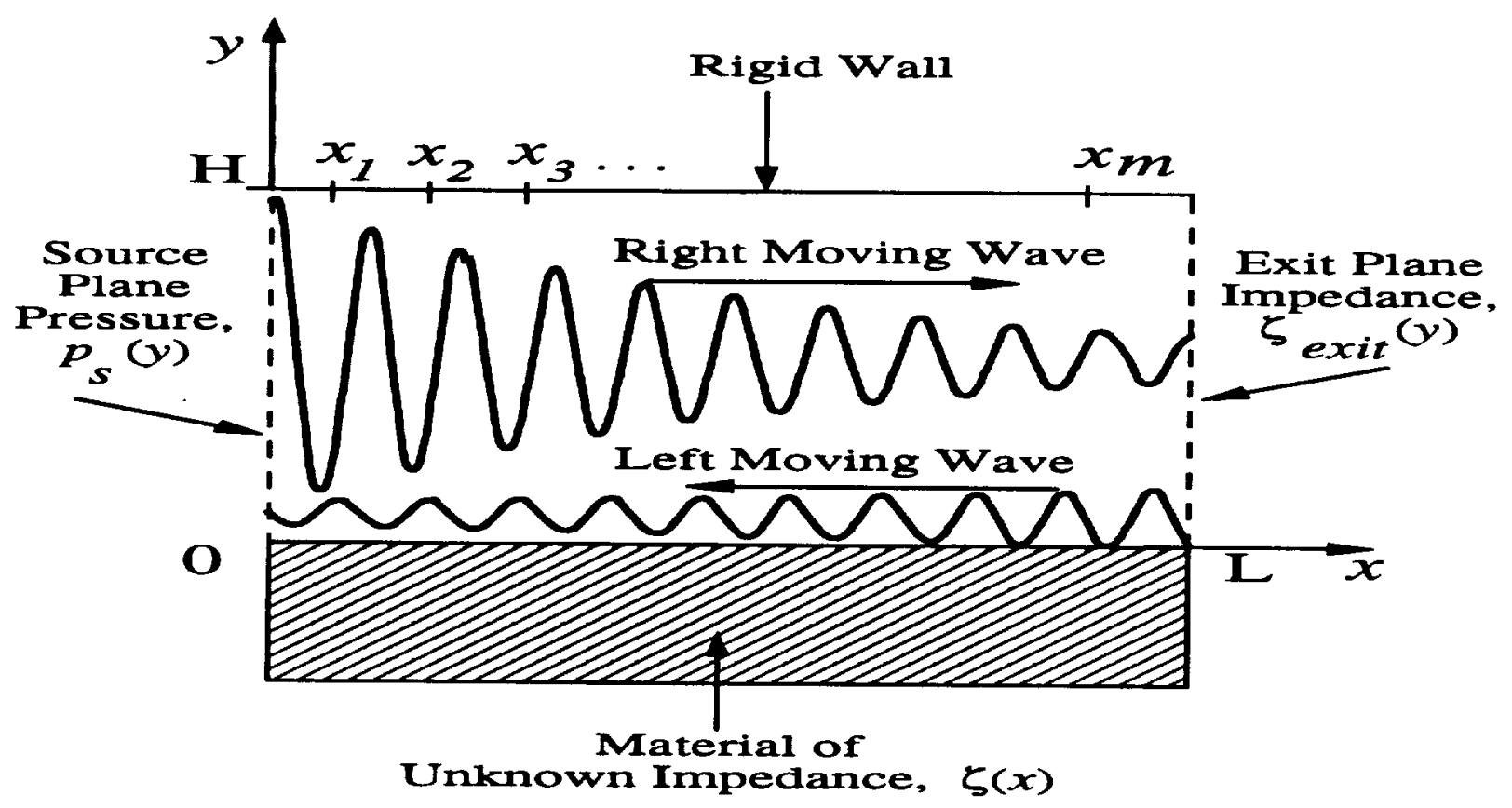

Figure 1: Two dimensional duct and coordinate system with a nonprogressive wave field

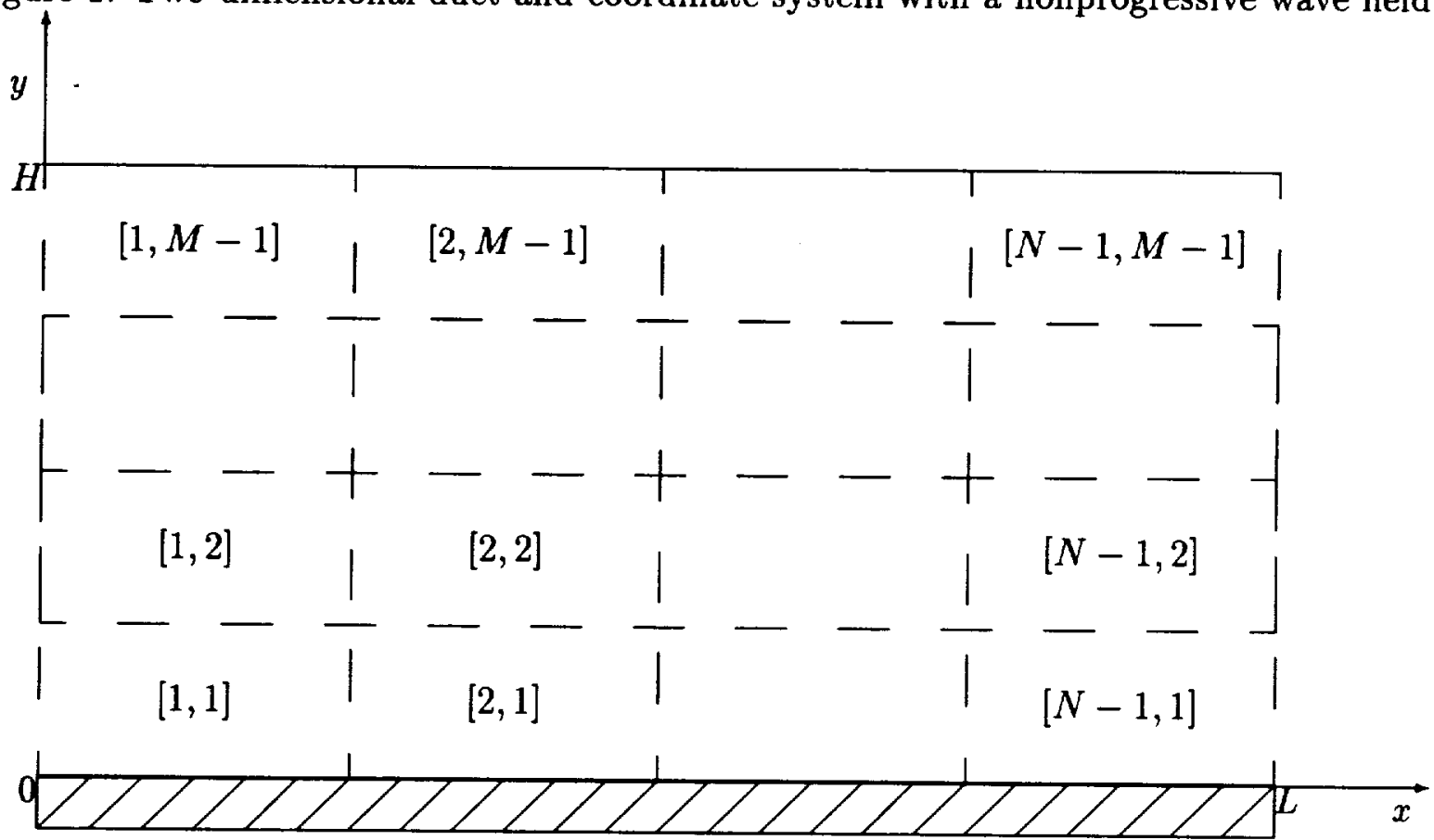

Figure 2: Finite element discretization of two dimensional duct 


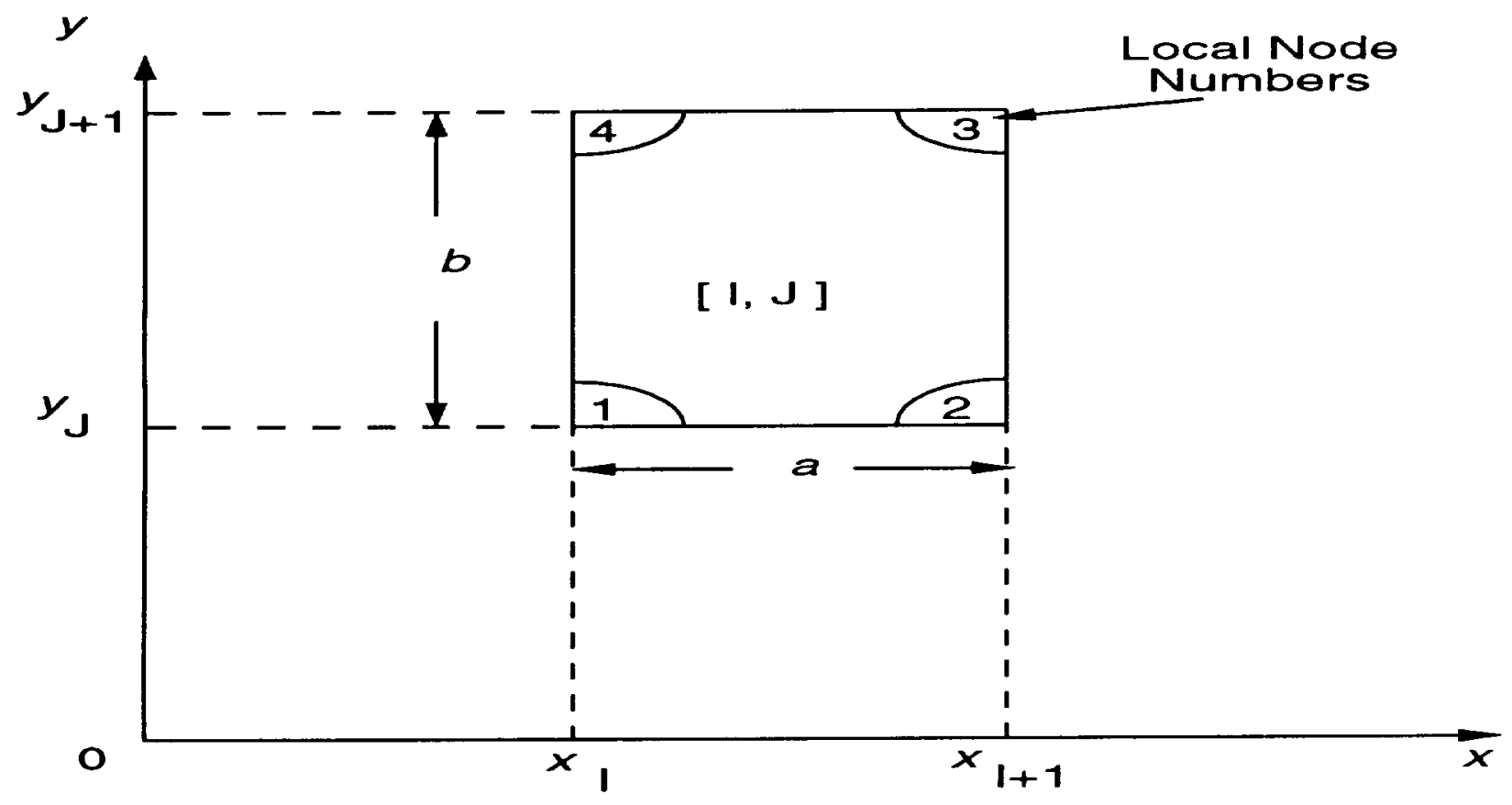

Figure 3: A typical finite element, $[I, J]$, and local node numbering system 
Structure of the global stiffness matrix with major blocks, $A_{I}, B_{I}$ and $B_{I}^{T}$

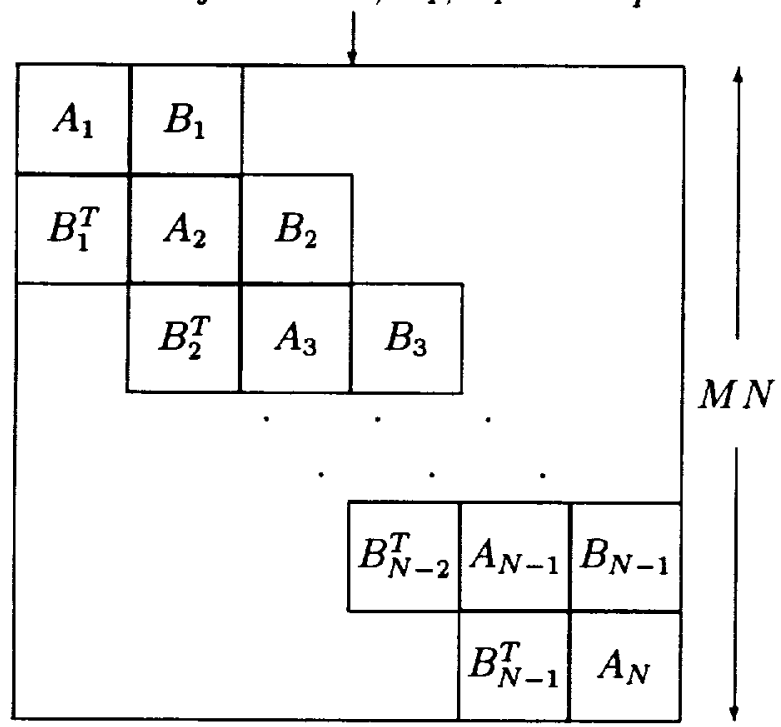

Structure of each major block, each $x$ is a complex number

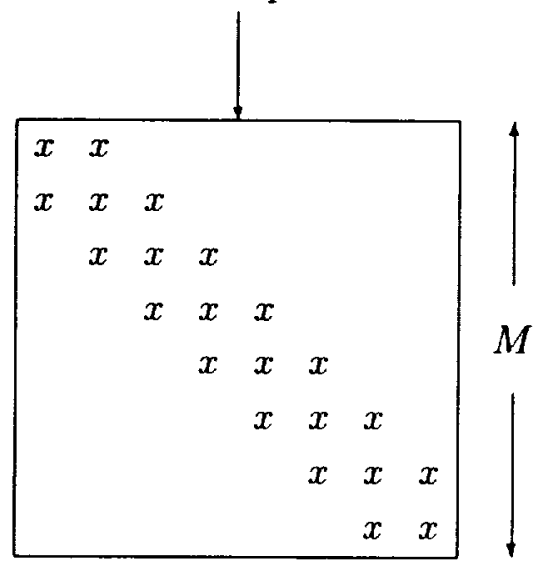

Figure 4: Structure of the global matrix and major blocks 


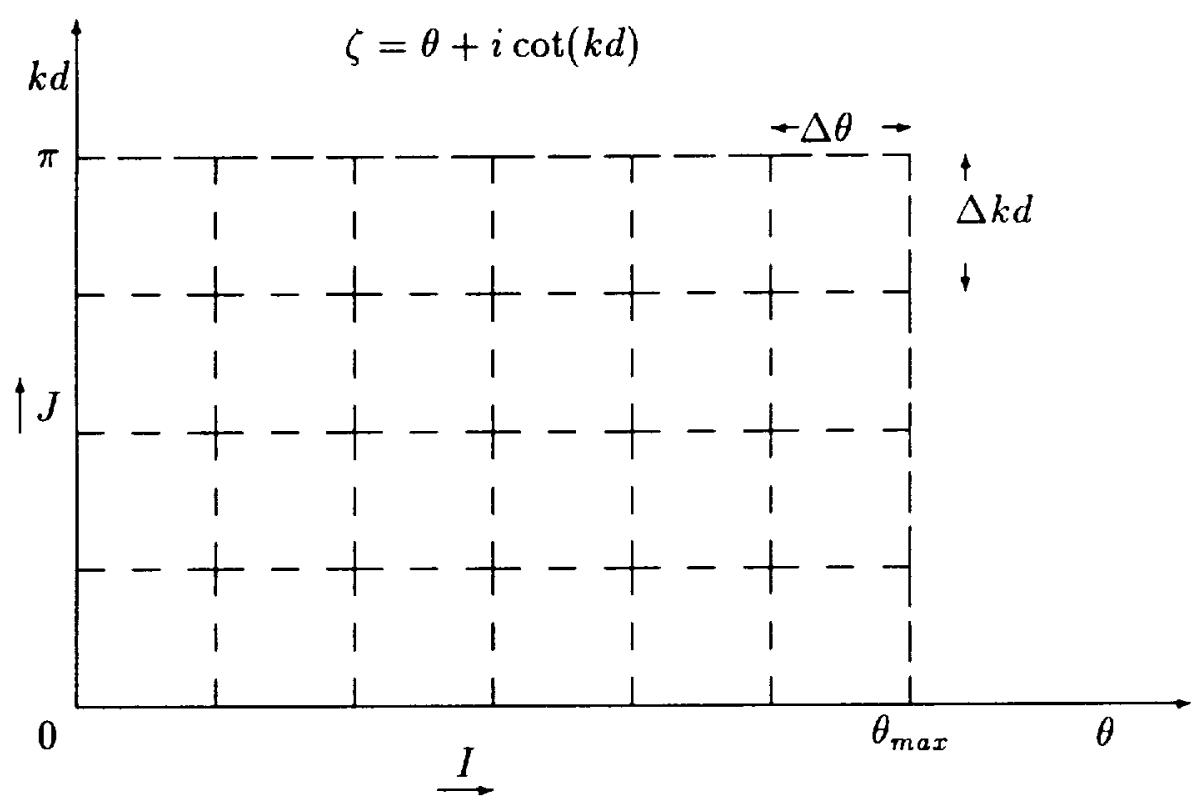

Figure 5: Impedance grid in the complex plane 


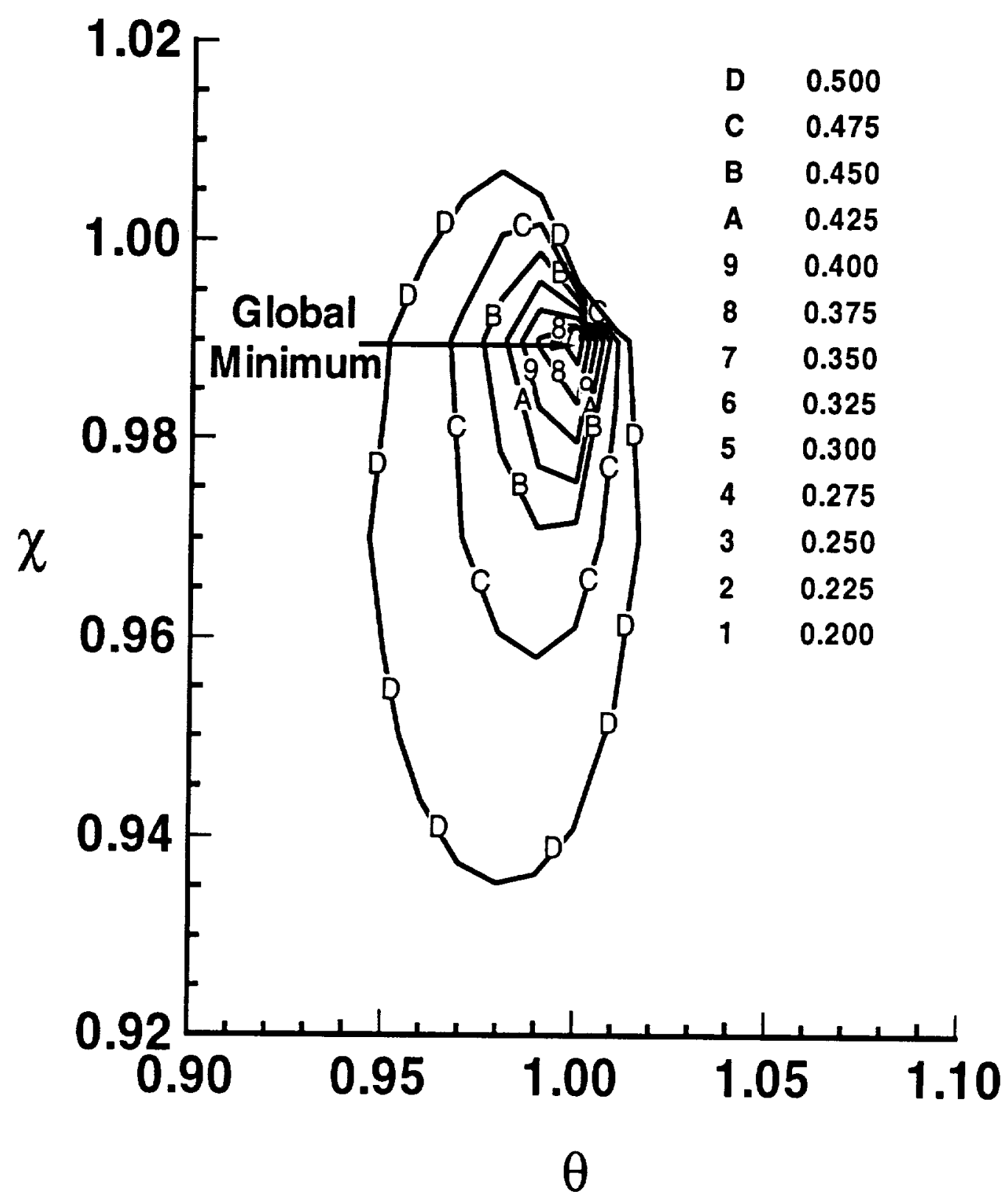

Figure 6: Contour plots of the wall error function, $E W(\zeta)$, at 500 Hertz (fine grid) 


\section{- Known value}

- Predicted value without random error

$\nabla$ Predicted value with random error

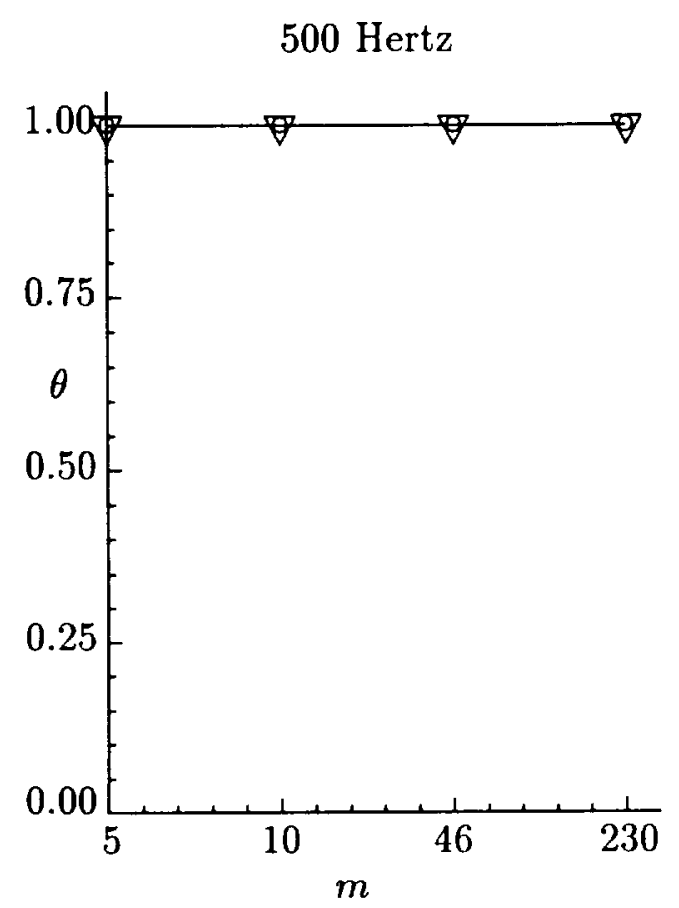

500 Hertz

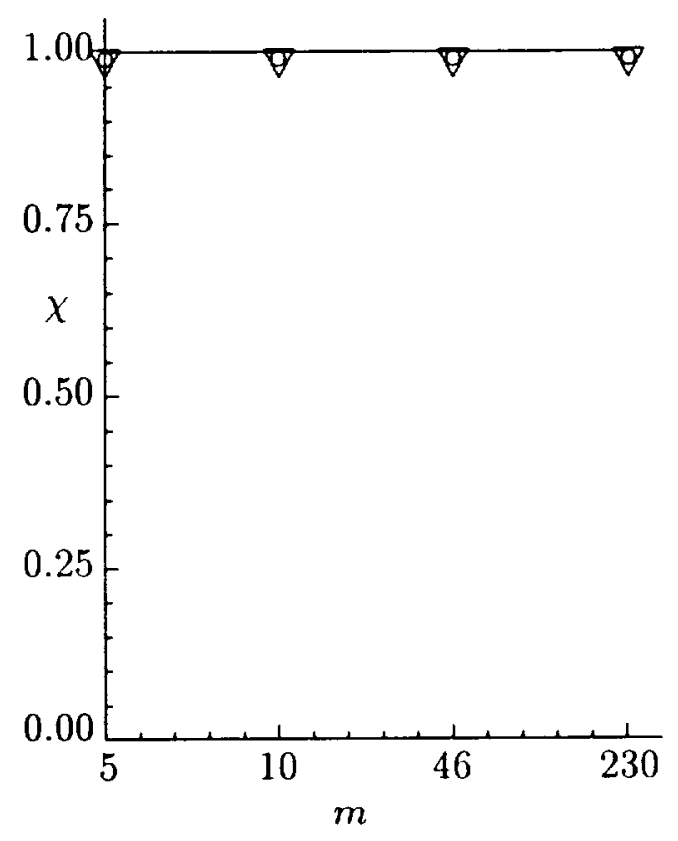

3,000 Hertz

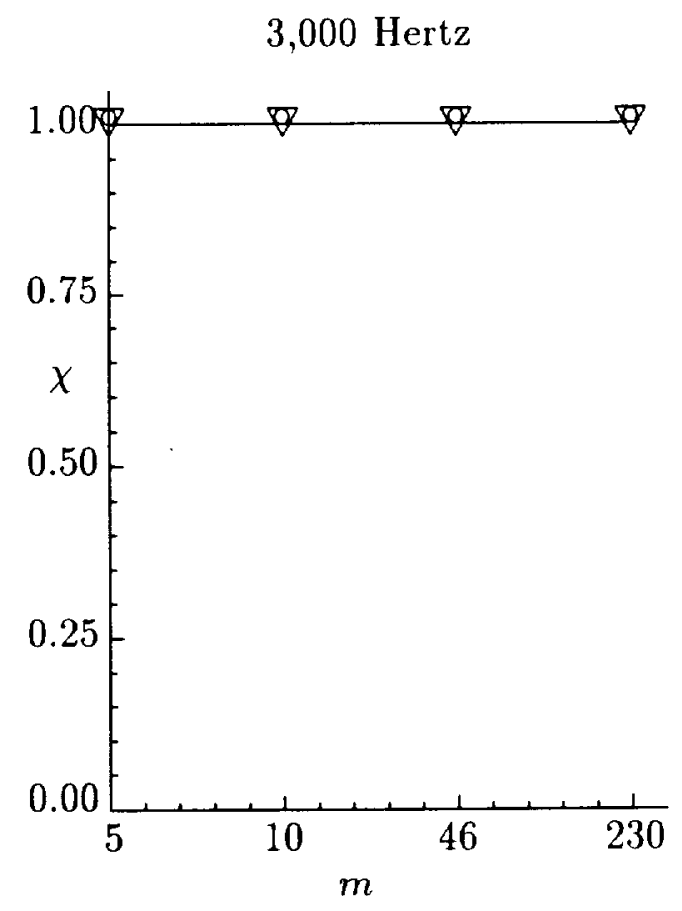

Figure 7: Resistance and reactance for the first material $(\zeta=\theta+i \chi=1+1 i)$ 
- Known value

- Predicted value without random error

$\nabla$ Predicted value with random error
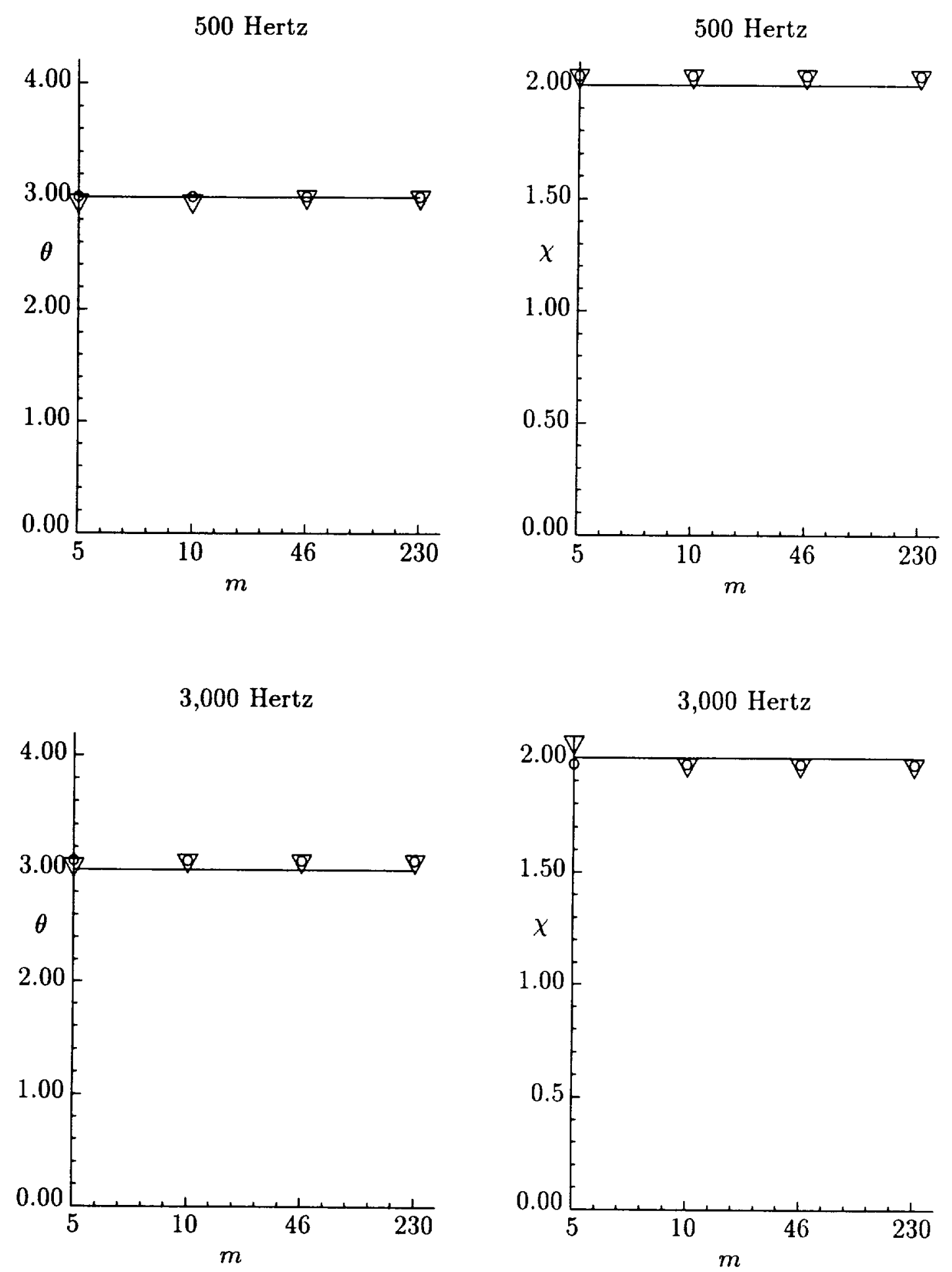

Figure 8: Resistance and reactance for the second material $(\zeta=\theta+i \chi=3+2 i)$ 
_ Known value

- Predicted value without random error

$\nabla$ Predicted value with random error

500 Hertz

500 Hertz
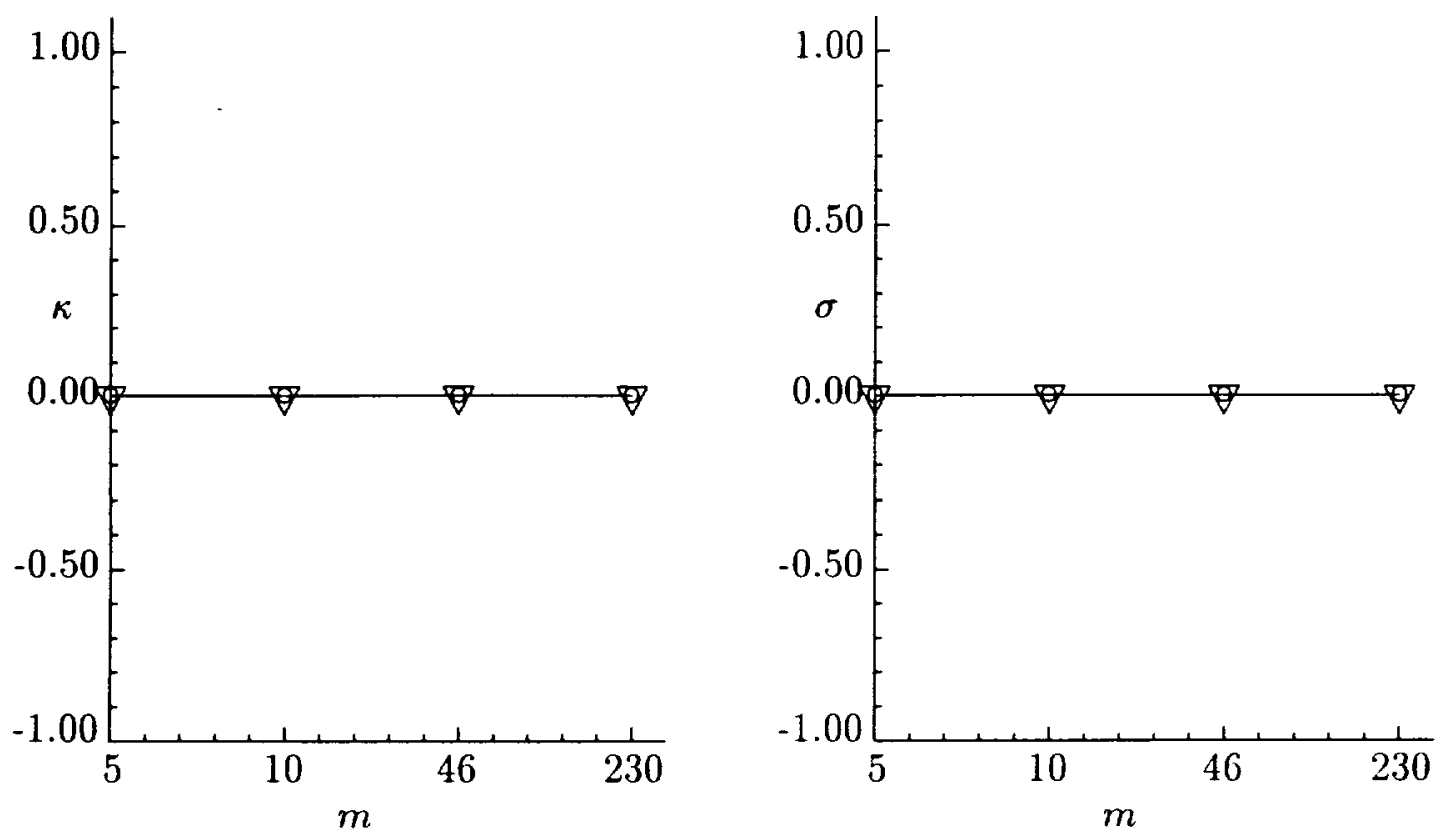

3,000 Hertz

3,000 Hertz
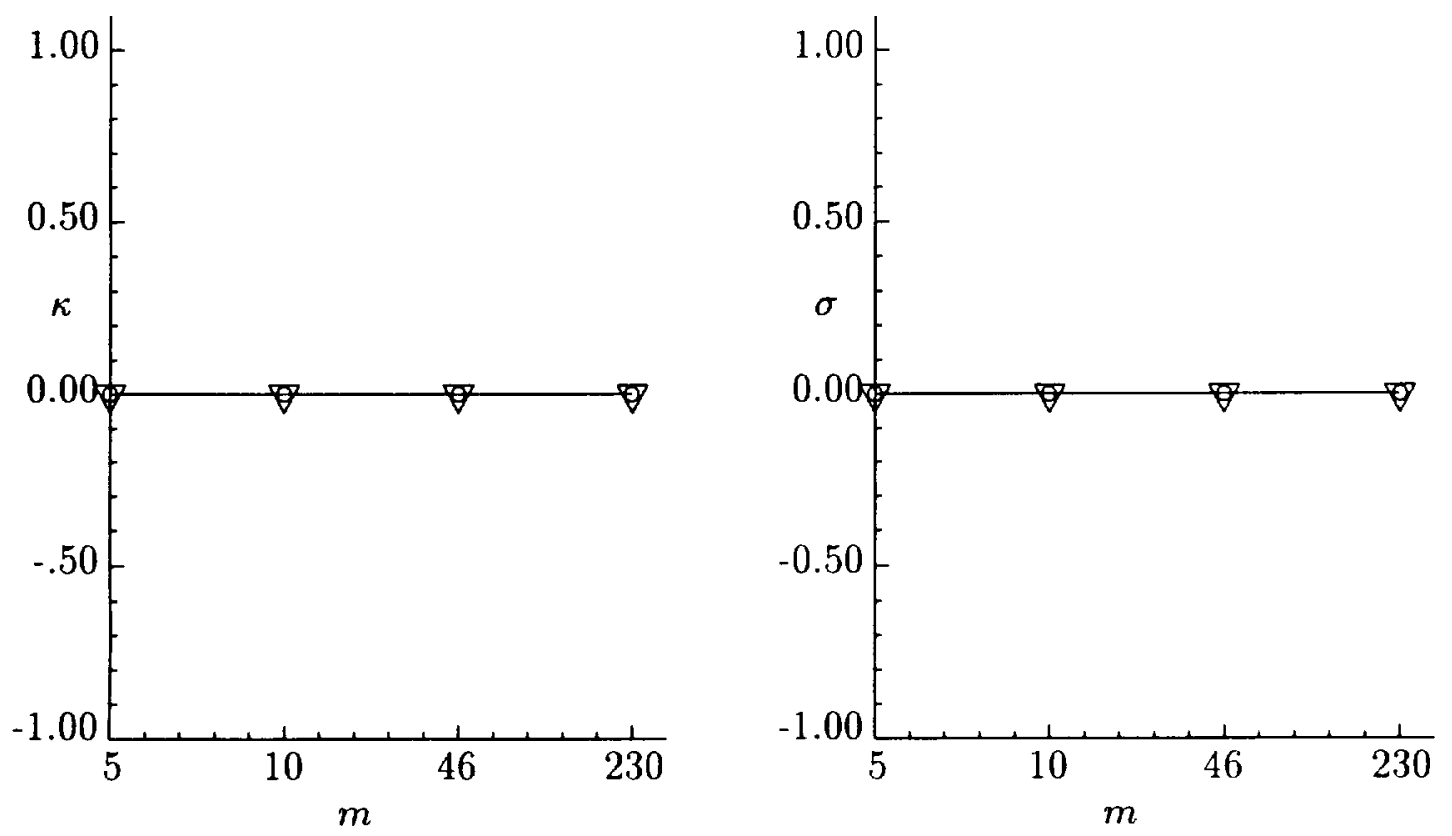

Figure 9: Susceptance and conductance for the third material $\left(\beta=\frac{1}{\zeta}=\kappa+i \sigma=0+0 i\right)$ 
Public reporting burden for this collection of inlormation is eatimated to average 1 hour per response. induding the timb for reviewing instructions, saarching oxisting data sources. gatheriog and maintaining the data nowded, and completing and reviewing the collection of information. Send comments regarding this burden eatimate or my other alped of thi

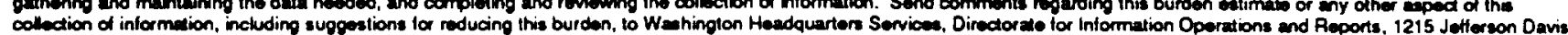
Highway, Suite 1204, Arlington, VA 22202-4302, and to the Oftice of Managoment and Budget. Paporwork Reduction Project (0704-0188), Waehington. DC 20503.

\begin{tabular}{|l|l|l|}
\hline 1. AGENCY USE ONLY (Laivo blank) & $\begin{array}{c}\text { 2. REPORT OATE } \\
\text { April } 1995\end{array}$ & $\begin{array}{c}\text { 3. AEPOAT TYPE AND DATES COVERED } \\
\text { Technical Memorandum }\end{array}$ \\
\hline
\end{tabular}

\section{TITLE AND SUBTITLE}

A Finite Element Propagation Model for Extracting Normal Incidence Impedance in Nonprogressive Acoustic Wave Fields

\section{FUNDING NUMBERS}

$537-02-22-02$

\section{AUTHOA(S)}

Willie R. Watson, Michael G. Jones

Sharon E. Tanner, Tony L. Parrott

7. PEAFORMING ORGANIZATION NAME(S) AND ADORESS(ES)
NASA Langley Research Center
Hampton, VA $23681-0001$
9. SPONSORING / MONITORING AGENCY NAME(S) AND ADDRESS(ES)
National Aeronautics and Space Administration
Washington, DC $20546-0001$

8. PERFORMING ORGANIZATION REPORT NUMBER

\section{SUPPLEMENTARY NOTES}

Willie R. Watson, Sharon E. Tanner, Tony L. Parrott: Langley Research Center

Michael G. Jones: Lockheed Engineering and Sciences Company

12. DISTAIBUTION I AVAILABILITY STATEMENT
Unclassilied-Unlimited
Subject Category 71

\section{ABSTRACT (Maximum 200 words)}

A propagation model method for extracting the normal incidence impedance of an acoustic material installed as a finite length segment in a wall of a duct carrying a nonprogressive wave field is presented. The method recasts the determination of the unknown impedance as the minimization of the normalized wall pressure error function. A finite element propagation model is combined with a coarseffine grid impedance plane search technique to extract the impedance of the material. Results are presented for three different materials for which the impedance is known. For each material, the input data required for the prediction scheme was computed from modal theory and then contaminated by random error. The finite element method reproduces the known impedance of each material almost exactly for random errors typical of those found in many measurement environments. Thus, the method developed here provides a means for determining the impedance of materials in a nonprogressive wave environment such as that usually encountered in a commercial aircraft engine and most laboratory settings.

\begin{tabular}{|c|c|c|c|}
\hline \multirow{2}{*}{$\begin{array}{l}\text { 14. SUBJECT TEAMS } \\
\text { finite elements } \\
\text { 2-D Helmholtz equation } \\
\text { impedance prediction }\end{array}$} & \multirow{2}{*}{\multicolumn{2}{|c|}{$\begin{array}{l}\text { nonprogressive wave fields } \\
\text { duct liners } \\
\text { tridiagonal solvers }\end{array}$}} & $\begin{array}{l}\text { 15. NUMBER OF PAGES } \\
20\end{array}$ \\
\hline & & & $\begin{array}{l}\text { 16. PRICE CODE } \\
\text { A03 }\end{array}$ \\
\hline $\begin{array}{l}\text { 17. SECUATTY CLASSIFICATION } \\
\text { OF REPORT } \\
\text { unclassified }\end{array}$ & $\begin{array}{l}\text { 18. SECUAITY CLASSIFICATION } \\
\text { OF THIS PAGE } \\
\text { unclassified }\end{array}$ & $\begin{array}{l}\text { 19. SECUAITY CLASSIFICATION } \\
\text { OF ABSTRACT } \\
\text { unclassified }\end{array}$ & $\begin{array}{l}\text { 20. UMITATION OF AESTRACT } \\
\text { unlimited }\end{array}$ \\
\hline \multicolumn{3}{|l|}{ NSN $7540-01-280-5500$} & $\begin{array}{l}\text { Standard Form } 298 \text { (Rev. 2-89 } \\
\text { Proworibed by ANSI Sid. Z39- 18 } \\
298-102\end{array}$ \\
\hline
\end{tabular}

